\title{
Teorías subjetivas en docentes sobre el aprendizaje y desarrollo socioemocional: Un estudio de caso
}

Cuadra Martínez, David Jorge; Salgado Roa, Jorge Alejandro; Lería Dul\#i\#, Francisco José; Menares Ossandón, Néstor Dante

Teorías subjetivas en docentes sobre el aprendizaje y desarrollo socioemocional: Un estudio de caso ${ }^{1}$

Revista Educación, vol. 42, núm. 2, 2018

Universidad de Costa Rica, Costa Rica

Disponible en: http://www.redalyc.org/articulo.oa?id=44055139014

DOI: https://doi.org/10.15517/revedu.v42i2.25659

Esta obra está bajo una Licencia Creative Commons Atribución-NoComercial-SinDerivar 3.0 Internacional. 


\title{
Teorías subjetivas en docentes sobre el aprendizaje y desarrollo socioemocional: Un estudio de caso ${ }^{1}$
}

\section{Subjective Theories on Teachers About Learning and Socio-Emotional Development: A Case Study}

David Jorge Cuadra Martinez [2]

Universidad de Atacama, Chile

DOI: https://doi.org/10.15517/revedu.v42i2.25659

david.cuadra@uda.cl

Jorge Alejandro Salgado Roa [3]

Universidad de Atacama, Chile

jorge.salgado@uda.cl

Francisco José Lería Dulčić [4]

Universidad de Atacama, Chile

francisco.leria@uda.cl

Néstor Dante Menares Ossandón [5]

Universidad de Atacama, Chile

nestor.menares@uda.cl

\author{
Redalyc: http://www.redalyc.org/articulo.oa?id=44055139014
}

Recepción: 15 Julio 2016

Aprobación: 17 Abril 2018

\section{RESUMEN:}

Con el objeto de describir e interpretar las teorías subjetivas (TS) acerca del aprendizaje y desarrollo socioemocional estudiantil, que presenta el profesorado de escuelas públicas de educación básica y secundaria de la región de Atacama, Chile, se realizó un

\section{NotAS DE AUTOR}

[2] Psicólogo, Magíster en Psicología. Desde el año 2010 ha adjudicado proyectos de investigación científica (fondos nacionales) en el área de psicología de la educación y desde un enfoque cognitivo, estudiando la génesis, desarrollo y cambio de teorías subjetivas. Actualmente académico del Dpto. de Psicología, Universidad de Atacama, Chile. Algunas de sus publicaciones son: Cuadra, D. (2016). Cambio representacional en los padres: reconstrucción de teorías subjetivas de la prevención del consumo de drogas en los hijos e hijas. Revista Estudios Pedagógicos, 42(1), 283-298; Cuadra, D. y Catalán, J. (2016). Teorías subjetivas en profesores y su formación profesional. Revista Brasileira de Educação, 21(65), 299-324; Cuadra, D., Castro, P. J., Vystrčilová, P. \& Jancic-Mogliacci, R. (2017). A review of research on teacchers' subjective theories: contributions to the study of teacher education. Psychology and Education Journal, 54(3), 1-22; Cuadra, D., Castro, P. J. y Juliá, Ma. T. (2018). Tres saberes en la formación profesional por competencias: integración de teorías subjetivas, profesionales y cientificas. Formación Universitaria, 11(5).

[3] Psicólogo, Doctor en Ciencias de la Educación. Actualmente subdirector y académico del Departamento de Psicología de la Universidad de Atacama, Chile. Participante activo como investigador responsable y co-investigador en proyectos con financiamiento nacional en la línea de psicología de la educación y calidad de vida laboral en contextos organizacionales. Publicaciones: Salgado, J. (2009). Pensamiento del profesor acerca del éxito o fracaso de su respectiva unidad educativa. REICE. Revista Iberoamericana sobre Calidad, Eficacia y Cambio en Educación, 7(3), 47-68; Lería Dulčić, F., Salgado, J., Almonte, I., Vega, M. \& Véliz, M. (2016). Prácticas sociales en torno a la inclusión de la discapacidad sensorial en escuelas públicas de Copiapó de Chile. Páginas de Educación, 9(1), 120-145; Leria, F. y Salgado, J. (2016). Estrés post-traumático y estrés subjetivo en estudiantes universitarios tras aluvión de barro. Revista Ciencias Psicológicas, 10(2),129-141; Salgado-Roa, J., Lería-Dulčić, F., Franco, P., Morales, R. \& Olivares, V. \& Gajardo, X. (2017). Efecto de la motivación sobre la profundidad en los procesos de estudio en universitarios de formación en pedagogía. REICE - Revista Iberoamericana sobre Calidad, Eficacia y Cambio en Educación, 15(4), 87-105. ORCID ID: 0000-0003-2470- 4988.

[4] Psicólogo, Magister en Psicología Clínica. Ha ejercido cargos en proyectos adjudicados municipales y regionales para el desarrollo de la atención plena en estudiantes, labores profesionales en centros sociales, escuelas y colegios, hogares de rehabilitación infantil y juvenil; junto a práctica privada en psicoterapia individual de integración. Actualmente en cargo a planta como académico del Dpto. de Psicología, Facultad de Humanidades de la Universidad de Atacama, Chile. Âreas de interés académico: educación contemplativa y áreas afines al bienestar y estrés subjetivo, satisfacción vital. Con varias publicaciones en estas áreas.

[5] Psicólogo, académico de la Universidad de Atacama. Desde el año 2014 director del Departamento de Psicología de la misma Universidad. Iniciándose recientemente en investigación universitaria, es responsable y co-investigador en proyectos con financiamiento interno. Publicaciones: Gomez, T. y Menares, N. (2013). Los aportes de paradigmas complejos y constructivistas para la enseñanza de la ciencia jurídica. Revista lus et Praxis, 20(1), 199-220. 
estudio descriptivo interpretativo, basado en el uso de metodología cualitativa y un diseño de estudio de caso. El muestreo fue de tipo intencionado y quedó constituido por 19 docentes de 2 escuelas de enseñanza básica y 1 establecimiento de educación primaria y secundaria, quienes se seleccionaron con el criterio de representatividad de casos. Se aplicaron 8 entrevistas episódicas y 2 grupos de discusión. Los datos se analizaron mediante la técnica de análisis de contenido de la teoría fundamentada y análisis específico para TS. Se encontró (a) un significado subjetivo del aprendizaje y desarrollo socioemocional, que lo relaciona con un sentido de vida en el estudiantado, además de la enseñanza de valores, hábitos y habilidades de relación interpersonal; (b) factores sociales, familiares, escolares e individuales que el grupo participante asocia con este proceso y que actúa como (c) facilitadores y obstaculizadores; (d) un proceso de desarrollo socioemocional evolutivo del estudiantado, que se describe en cuatro fases y prácticas pedagógicas necesarias de adecuar frente a estos períodos; (e) propuestas para la educación socioemocional y (f) una caracterización de la formación docente para la educación socioemocional, que considera experiencias de aprendizaje formales e informales. En la discusión se analizan las características de las TS encontradas, su relación con las prácticas pedagógicas y la formación del profesorado.

Palabras Clave: Teorías subjetivas, educación socioemocional, desarrollo emocional, formación docente.

\section{Abstract:}

In order to describe and interpret subjective theories (ST) regarding student learning and socioemotional development, an interpretative descriptive study based on a qualitative methodology and case study design was carried out in a group of primary and secondary public school teachers in Atacama (Chile). A convenient sample constitituted by nineteen teachers from two primary schools and one primary and secondary education school were used under the case representativeness criteria. Eight episodic interviews and two discussion groups were made. Data was analyzed using the grounded theory content analysis technique with a specific analysis for ST. Results showed: (a) a subjective meaning of learning and emotional development, related to the teaching of values, habits, interpersonal skills and a sense of life in the students; (b) social, family, schooling and individual factors that teachers associate with this process acting as (c) facilitators and obstacles; (d) a process of evolutionary student social-emotional development described in four phases and pedagogical practices necessary to adapt to face these periods; (e) proposals for socioemotional education; and $(\mathrm{f})$ a characterization of teacher training for social-emotional education considering experiences of both formal and informal learning. The characteristics of ST found as well as their relationship with pedagogical practices and teacher training are analyzed.

KEYWORDS: Subjective theories, social and emotional education, emotional development, teacher training.

Los estudios acerca de la eficacia educativa han presentado evidencias significativas de la relevancia que tiene la formación del profesorado en los resultados de aprendizaje, existiendo actualmente una importante producción de investigaciones, programas y orientaciones que apuntan a mejorar su formación profesional (Bruns y Luque, 2014; Friesen, 2009; Fullan, 2002; García y Ballesteros, 2003).

Con respecto a la educación socioemocional, la figura docente aparece como clave (Díaz, 2014; Ramírez, 2011), en la medida que la escuela constituye el espacio privilegiado para la formación ciudadana y que la enseñanza transcurre sobre una determinada convivencia escolar, que implica un potencial para aprender a vivir en sociedad, por lo que la educación en valores (Sandoval, 2014) y la socioemocional (Castro, 2006) pasan a ser pilares del proceso formativo del alumnado.

Sin embargo, algunos estudios dan cuenta de debilidades en la formación del profesorado para la educación socioemocional (Ochoa y Peiró, 2012a), pues en las universidades se privilegia la preparación para el desarrollo cognitivo, en desmedro de la adquisición de competencias para la formación social, valórica y emocional del alumnado (Díaz, 2014; López de Lérida, 2009). Se propone que esta formación esté orientada a favorecer, en el personal docente, la capacidad de reflexión sobre su actuar ético y a comprender el desarrollo social y emocional del estudiantado (Ochoa y Peiró, 2012b). Al respecto, actualmente se entiende la educación socioemocional como una "condición primaria para el despliegue de la personalidad" (García, 2012, p. 25), que consiste en el desarrollo de competencias sociales y emocionales que permitan el establecimiento de relaciones interpersonales positivas, la valoración personal, del medio social y natural (Berger, Alcalay, Torretti \& Milicic, 2011), lo que redunda en un aumento de la capacidad para conocerse a 
sí y a las demás personas y para resolver problemas con creatividad (Berger, Milicic, Alcalay, Torretti y Arab, 2009; Cohen, 2003).

Desde lo anterior, la preocupación que muestre la escuela, especialmente el cuerpo docente, por el desarrollo personal de sus estudiantes actúa como un factor moderador del desarrollo socioafectivo del niño o niña o la aparición de conductas antisociales (Díaz, 2014). Por lo que se espera en el profesorado competencias para manejar relaciones interpersonales y regular el desarrollo emocional de sus estudiantes (Vivas, Chacón y Chacón, 2010), debiendo conjugar su propia teoría de la mente o representación subjetiva sobre el aprendizaje y desarrollo socioemocional, con el conocimiento experto acerca del tema. Esto, a propósito de que la formación docente no está restringida al saber científico técnico aprendido, sino que también depende de experiencias formativas que ocurren en contextos informales de aprendizaje antes, durante y posteriormente a la formación inicial, desde donde se adquiere un tipo de saber que es intuitivo o subjetivo, que regula en algún nivel sus prácticas pedagógicas (Cuadra y Catalán, 2016; Hindin, 2010).

Algunos estudios que abordan la subjetividad del profesorado en el aprendizaje y desarrollo emocional han develado que consideran tener una insuficiente formación inicial para abordar la temática, además de una mayor presencia de emociones displacenteras que placenteras respecto de su rol profesional (De Leyva, 2015; Vivas, Chacón y Chacón, 2010).

En un estudio realizado en una comuna de Santiago de Chile y en docentes de enseñanza básica (Toro y Berger, 2012), se indagó cómo este grupo profesional representa subjetivamente su rol en la educación socioemocional, encontrando que se define como una figura de apego sencundaria para el estudiantado, que se concreta en la implementación de roles parentales que producen conflictos en su desempeño profesional. Profundizando en la temática, Kington (2012) estudió las narrativas de docentes del Reino Unido, acerca de la relación que establecen con sus estudiantes a lo largo de su carrera profesional. Encontró cinco áreas desde las cuales describe este vínculo: con base en la interacción, el comportamiento, las expectativas, la proximidad y el control. Además, observó que el acento puesto en estas áreas varía a través de la carrera profesional docente $y$, en ocasiones, produce tensiones en la relación con el estudiantado. Otros estudios se han focalizado en las explicaciones que presentan docentes sobre algún ámbito relacionado con el desarrollo socioemocional del alumnado, por ejemplo, acerca de la motivación y expectativas de éxito y fracaso escolar que presentan los cuerpos docentes (Castro et al., 2012), la violencia escolar, desde las explicaciones de docentes en cargos directivos (Von Reininghaus, Castro y Frisancho, 2013) o el rol que tiene el profesorado en la convivencia escolar (Retuert y Castro, 2017).

De esta manera, una forma en que el personal docente puede representar subjetivamente el aprendizaje y desarrollo socioemocional es mediante teorías subjetivas (TS). Desde este programa de investigación (Catalán, 2010, 2016; Flick, 2006), se asume que las TS integran el conocimiento profesional de las profesoras y los profesores (Castro y Cárcamo, 2012; Catalán, 2011; Cuadra, 2009; Cuadra y Catalán, 2016; Cuadra, Jorquera y Pérez, 2015) y corresponden a un tipo de representación argumentativa (cognitiva o subjetiva) de mayor complejidad (Flick, 2006), elaboración y generalidad; ocupan un lugar importante en las representaciones que el profesorado ha construido de lo educacional (Janssens y Kelchtermans, 1997), regulan en algún nivel sus prácticas pedagógicas, sus creencias previas y el proceso de cambio (Castro, Agüero, Barraza, Escobar y Jorquera, 2012); por lo que la investigación de la subjetividad de la persona mediante TS ha sido considerada como una opción metodológica más coherente (Flick, 2006) y un paradigma emergente de investigación educativa (Cuadra, Castro, Vystrčilová \& Jancic Mogliacci, 2017).

En función de lo anterior, parece congruente preguntarse: ¿cómo explican docentes el aprendizaje y desarrollo socioemocional de sus estudiantes?, ¿cómo se construyen estas TS y cuál es su relación con sus prácticas pedagógicas? Y, según su juicio: ¿cómo se favorece el aprendizaje y desarrollo socioemocional en el estudiantado y cómo se obstaculiza? De esta manera, se ha definido el siguiente problema de estudio: ¿Cuáles son y cómo se construyen las teorías subjetivas acerca del aprendizaje y desarrollo socioemocional de sus estudiantes, que presentan docentes de tres escuelas públicas de enseñanza básica y secundaria, de una 
comuna de la región de Atacama? Como objetivo general, se buscó describir e interpretar estas TS y a nivel específico (a) describir el sentido subjetivo que le otorgan al aprendizaje y al desarrollo socioemocional de sus estudiantes; (b) determinar la manera en que explican el aprendizaje y el desarrollo socioemocional de sus estudiantes; (c) caracterizar las propuestas que consideran que promueven la enseñanza y el desarrollo socioemocional en sus estudiantes; (d) determinar la forma en que se construyen estas TS y (e) analizar cómo se relacionan estas TS con sus prácticas pedagógicas.

En cuanto a la pertinencia y relevancia de este estudio, es necesario señalar que la educación socioemocional no solo es fundamental para lograr el éxito académico del estudiantado, sino que también lo es para la construcción de una sociedad más justa y democrática (Berger et al., 2009), por lo que se requiere de investigaciones que permitan orientar la forma en que se debe desarrollar esta competencia en el personal docente (Díaz, 2014; López de Lérida, 2009; Ochoa y Peiró, 2012b), sobre todo a partir de la subjetividad de este mismo (Salgado, 2009) y desde las TS, por tener estas una incidencia importante en las prácticas educativas (Catalán, 2011; Castro et al., 2012; Cuadra et al., 2015; Donovan, Borda, Hanley y Landel, 2015).

\section{MÉTODo}

\section{Tipo de estudio, metodología y diseño}

Corresponde a un estudio descriptivo interpretativo, basado en el uso de metodología cualitativa, por ser esta la que permite acceder a la subjetividad del participante de manera holista, humanista y desde su propia perspectiva (Flick, 2006; Taylor y Bogdan, 1987). El diseño corresponde a un estudio de caso (Burgos, 2011). "El estudio de casos es el estudio de la particularidad y de la complejidad de un caso singular, para llegar a comprender su actividad en circunstancias importantes" (Stake, 1999, p. 11). De esta manera, el caso es representativo de lo que se está investigando. En este trabajo, fue conveniente estudiar a docentes de los establecimientos educativos señalados en la muestra, a fin de comprender de manera profunda y detallada la forma en que explican el aprendizaje y desarrollo socioemocional de sus estudiantes. Además, el diseño se enmarca en el programa de investigación de TS (Castro y Cárcamo, 2012; Catalán, 2010, 2011; Cuadra y Catalán, 2016; Flick, 2006; Groeben \& Scheele, 2000), que asume que este tipo de representaciones subjetivas le permiten a la persona explicar su propio comportamiento y el mundo, actuando como un regulador de sus acciones y de otro tipo de representaciones.

\section{Participantes}

La muestra corresponde a 19 docentes de dos escuelas de educación pública primaria y una que imparte educación pública primaria y secundaria, ubicadas en la región de Atacama, Chile. Se seleccionaron mediante un muestreo intencionado de tipo opinático (Ruiz, 1999) y con el criterio de representatividad de casos. Se consideraron docentes que imparten distintas asignaturas, de ambos sexos (44\% varones y $56 \%$ mujeres) y presentan distintos años de servicio profesional. Al respecto, al momento de la aplicación de los instrumentos presentaron un promedio de edad de 43 y 17 años de servicio profesional.

Como criterios de inclusión se consideró: (a) estar en posesión de título profesional de profesor, (b) haber ejercido la docencia por lo menos 2 años y (c) ser parte del plantel docente del establecimiento educativo. Las instituciones educativas se caracterizan por ofertar educación primaria y secundaria en la región, a un grupo socioeconómico medio a medio alto, con resultados en el Sistema de Medición de la Calidad de la Educación (SIMCE) similares a establecimientos de igual nivel socioeconómico. En Chile, la prueba SIMCE evalúa los resultados de aprendizaje de los establecimientos educativos, en diferentes asignaturas o ámbitos de aprendizaje. 


\section{Procedimiento de recolección de información}

Las personas participantes se contactaron a través del equipo directivo de los establecimientos educativos. En instalaciones de las escuelas y liceo, se procedió a informar al personal docente los fines de la investigación y los criterios éticos, solicitándole su participación mediante la firma de un consentimiento informado. Posteriormente, en un horario acordado con docentes y equipo directivo, se aplicaron en el mismo establecimiento entrevistas episódicas y grupos de discusión. La indagación se inició con las entrevistas episódicas, para posteriormente implementar los grupos de discusión, opción metodológica que apuntó a lograr mayor credibilidad, a partir de la posibilidad de contrastar y profundizar los datos obtenidos con las entrevistas episódicas, en un contexto de trabajo colectivo, favorecido por los grupos de discusión (Catalán, 2010). La recolección de los datos se realizó en un período aproximado de 3 meses.

\section{Instrumentos}

a) a) Entrevista episódica: Se aplicaron 8 entrevistas episódicas. Se optó por este instrumento, dado que permite acceder a un contenido narrativo-episódico y semántico de la persona, en este caso, del problema de estudio, facilitando así la producción de TS en las personas participantes (Flick, 2006). Para su implementación se utilizó un guión temático que consideró los siguientes ejes de indagación: (a) significado y caracterización de aprendizaje y desarrollo socioemocional estudiantil; (b) factores que se asocian con el aprendizaje y desarrollo socioemocional estudiantil; (c) formación docente y aprendizaje y desarrollo socioemocional estudiantil y (d) sugerencias para el aprendizaje y desarrollo socioemocional estudiantil. Su aplicación se basó en la propuesta de Flick (2006), que estimula en las personas participantes (a) la narración de situaciones y de cadenas de situaciones relacionadas con el tema de estudio, en este caso, la narración de experiencias de participantes asociadas a la educación socioemocional (por ejemplo: "¿me podría contar una experiencia desde la cual haya aprendido a educar socioemocionalmente a sus estudiantes?"); (b) supuestos sobre el tema, por ejemplo, con episodios hipotéticos sobre la educación socioemocional propuestos por quien investiga, con base en preguntas tales como: "¿qué harías si...?", "suponiendo que... ¿cómo explicarías tal cosa?"; (c) definiciones subjetivas sobre tópicos del tema, con preguntas orientadas a determinar el significado y la caracterización de ámbitos de la educación socioemocional (por ejemplo: "¿Qué es para ti educar socioemocionalmente?"); (c) establecimiento de relaciones abstractas, a fin de lograr explicaciones sobre el tópico estudiado (por ejemplo: "¿Cómo relacionas tu proceso formativo con la educación socioemocional que impartes a tus estudiantes?"). Cada entrevista tuvo una duración aproximada de 1 hora y fue grabada en audio.

b) b) Grupo de discusión: Se aplicaron 2 grupos de discusión constituidos cada uno por entre 5 y 6 docentes que no habían participado en la fase de entrevistas: uno de estos en uno de los establecimientos de educación primaria y el otro, en el de educación secundaria. Para su implementación, se utilizó el mismo guion temático de las entrevistas y la técnica de Flick (2006) de indagación de episodios biográficos (narración de situaciones y de cadenas de situaciones relacionadas con el tema de estudio, supuestos sobre el tema, definiciones subjetivas sobre tópicos del tema y establecimiento de relaciones abstractas), dado que se ha visto la factibilidad de utilizar esta misma herramienta en los grupos de discusión (Cuadra, 2016). Para el registro de datos se procedió a grabar en audio, previo consentimiento de las personas participantes. La aplicación de este instrumento tuvo una duración aproximada de 1 hora. 


\section{Procedimiento de análisis de datos}

Los datos se analizaron utilizando el software Atlas.ti 6.0 y mediante la técnica de análisis de contenido de la grounded theory (Strauss \& Corbin, 2002), que consiste en reducir la información mediante el método comparativo constante y tres procesos de codificación. En primer lugar, se analizaron los datos obtenidos mediante las entrevistas episódicas y posteriormente los correspondientes a los grupos de discusión:

1. Codificación abierta: Se transcribieron las entrevistas y grupos de discusión a fin de elaborar códigos a partir de sus oraciones, frases o párrafos. Durante esta etapa, se identificaron TS explicitadas en el texto, mediante enunciados del tipo si, entonces, porque, esto conduce a aquello, etc. e implícitas, inferidas por el equipo investigador (Catalán, 2010, 2016; Fick, 2006). De esto resultó un libro de códigos.

2. Codificación axial: Los códigos obtenidos se sometieron a análisis a fin de agruparlos en categorías representativas de los objetivos, estableciendo consensos entre el equipo investigador. De esto resultaron seis categorías que son descritas en los hallazgos de este estudio: (a) el sentido subjetivo del aprendizaje y desarrollo socioemocional; (b) aprendizaje y desarrollo socioemocional: factores asociados; (c) obstaculizadores y facilitadores del aprendizaje y desarrollo socioemocional; (d) el desarrollo socioemocional como proceso; (e) propuestas formativas para el aprendizaje y desarrollo socioemocional y (f) la formación docente para el desarrollo socioemocional del alumnado.

3. Codificación selectiva: Finalmente, se identificó una categoría semántica como núcleo, a fin de establecer relaciones entre categorías, lo que permitió elaborar una teoría comprensiva del problema de estudio. En este caso, la categoría núcleo quedó representada por la TS del desarrollo socioemocional como proceso (Figura 1).

El procedimiento anterior se complementó con la propuesta de Catalán $(2010,2016)$. En este caso se analizó el nivel de elaboración que presentan las TS, con base en la capacidad explicativa del enunciado; el contenido de estas TS y la orientación a la acción que promueven.

\section{Resultados}

\section{El sentido subjetivo del aprendizaje y desarrollo socioemocional}

Para el personal docente, el aprendizaje y desarrollo socioemocional es una dimensión transversal del currículo que consiste en desarrollar en el estudiantado valores, hábitos y habilidades de relación interpersonal, a fin de que aprenda a convivir en sociedad. Algunos atributos de personalidad que este debería adquirir son: (a) perseverancia para lograr sus metas; (b) respeto en las relaciones interpersonales; (c) hábitos de cortesía; (d) honestidad; (e) disciplina y (f) buen desempeño en las tareas. Así lo expresan algunos profesores y profesoras:

Una dimensión que generalmente no está explícita, digamos, en el currículum o quizás se explicita pero siempre está enfocada digamos en los objetivos transversales (GI, PII $)^{6}$.

Entonces uno tiene que enseñarles hábitos, enseñarles a relacionarse entre ellos. . no siempre está preocupado en general de cómo el niño se va relacionando y para eso existe que trabajen en equipo, que sea respetuoso, que salude (EIV).

Asimismo, para parte del grupo de participantes, educar socioemocionalmente implica enseñar al estudiantado a reconocer sus emociones, expresarlas y regularlas, lo que además permite desarrollar un 
sentido de vida y bienestar subjetivo, constituyéndose en un requisito para el aprendizaje de los contenidos curriculares.

Tiene que ver con reconocerse en esa dimensión emocional y ver cómo deja fluir y en qué emoción está y cómo eso está influyendo en el comportamiento, en la forma en la que siento, en la forma en la que en este minuto pienso y desde ahí emprender un camino de dejar fluir eso y administrarla de tal manera que me permita seguir desarrollándome (GDI; PIII).

Espiritual, más que nada es como sentirse parte de una sociedad, sentirse útil, sentirse pleno en lo que uno está haciendo y dejar de lado ya cuánto va a ganar, "si te gusta esto, hazlo, porque tú eres feliz en esto", felicidad más que logro económico (EII).

\section{Aprendizaje y desarrollo socioemocional: factores asociados}

En general, este grupo de docentes considera que el aprendizaje y desarrollo socioemocional se produce por una serie de factores que se pueden clasificar en:

\section{Factores sociales}

Los niveles socioeconómico y sociocultural son considerados condicionantes del aprendizaje y desarrollo socioemocional, en la medida en que constituyen el contexto en donde el estudiantado aprende valores, hábitos y desarrolla características de personalidad. Para la mayoría de docentes, un nivel socioeconómico y cultural bajo limita las oportunidades de desarrollo y moldea comportamientos menos ajustados socialmente.

También tiene que ver mucho el tema sociocultural y lo sigo viendo en el tema del lugar donde ellos se desenvuelven y por qué lo derivo al nivel socioeconómico, porque generalmente si no contamos con los recursos necesarios, si no contamos con los recursos, obviamente vamos a llegar a un lugar que no es tranquilo para vivir, de alguna u otra manera sí puede afectar, pero también afecta mucho el tema cultural donde ellos se desenvuelven después de la jornada escolar (EIV).

\section{Factores individuales}

También atribuyen características de personalidad a estudiantes, pues condicionan la forma de adaptarse al entorno escolar. Dentro de esto, el rol de género tiene asociados atributos que le otorgan diferencias al funcionamiento escolar de hombres y mujeres, referidos a una mayor autonomía, sensibilidad y madurez en las mujeres o un menor grado de conflictos entre hombres. Estas características podrían ser constituyentes de los estereotipos asociados al rol de género, construidos por estos participantes. Así mismo, la perseverancia y un alto sentido de autoeficacia son indicados como atributos que, cuando se poseen, promueven el desarrollo personal.

Considero que las niñas son más delicadas, es mi parecer, mi apreciación, que si una niña tuviera un problema en comparación con un hombre, lo tomaría de forma diferente. Como digo en mi parecer, las niñas son como más de sentimiento, son más delicadas, son más cariñosas (EIV).

Cuando el niño tiene carencia en la casa, uno puede suplir algunas cosas, pero si el niño tiene ganas de hacer algo, tú ves que el niño se quiere superar, entonces son las ganas de ser algo (EII).

\section{Factores familiares}

Indican que la familia tiene un rol decisivo en el aprendizaje y desarrollo socioemocional del estudiantado. Consideran que aspectos como la disciplina, disposición al aprendizaje, valoración de la educación y resolución de conflictos interpersonales son resultantes de la crianza familiar y que, actualmente, este grupo presenta déficit en esta función. La carencia afectiva del estudiantado es considerada por este grupo 
participante como un déficit que puede redundar en que los alumnos y alumnas busquen resolver esta necesidad en la escuela.

Cuando uno es afectuoso en su casa, debiera demostrarse en ese niño y lo otro, cuando no tiene afecto en su entorno familiar busca el afecto (GDII; PIV).

Hay niños que tienen muchos recursos, reciben sueldos de la minería, pero falta una base más social y valórica en ellos, porque las familias valen de acuerdo al dinero que tienen y los valores, ya la base valórica de cómo respetar a las personas, no ser agresivo, eso se está perdiendo porque ya los padres trabajan, los niños están teniendo antivalores (EI).

\section{Factores escolares}

Señalan que el factor escolar más importante para la formación y desarrollo socioemocional del alumnado es el personal docente. Explican que el tipo de relación interpersonal que cada docente establece con sus estudiantes puede marcar la diferencia en la disposición al aprendizaje, la forma en que aprende a resolver los conflictos interpersonales, la apertura y aceptación de sugerencias y la disciplina. Asimismo, se reconoce que el personal docente constituye un modelo a seguir para el alumnado y que la motivación por su profesión puede determinar la presencia en clases de estudiantes que se aplican o desinteresan por aprender. Finalmente, consideran que la cultura escolar también incide en el desarrollo socioemocional, en la medida en que la comunidad educativa transmite valores y normas.

Porque uno marca a sus niños, los niños son muy parecidos a uno. Si el profesor es artístico, si el profesor es apático, si el profesor es medio flojito, medio lento, los niños son así, uno lo ve (EII).

Lo más importante para que el niño esté dispuesto a aprender, nuevamente yo creo que es la disposición del profesor (EIV).

La transformación de tipo psicológica, el apoyo del colegio, entonces va a depender mucho de cómo sea el ambiente la forma cómo se va a ir desarrollando, bueno y aquí en realidad siempre le prestamos harta atención a eso, queremos bastante a los alumnos (GDII; PIV).

En esta relación entre docente-estudiante, la herramienta de desarrollo socioemocional la constituye el diálogo, las experiencias biográficas emocionales que transcurren en la escuela y la reflexión sobre estas mismas, para lo cual se requiere de tiempos y espacios adecuados.

\section{Obstaculizadores y facilitadores del aprendizaje y del desarrollo socioemocional}

Se identifican una serie de factores que obstaculizan el aprendizaje y desarrollo socioemocional del estudiantado: (a) los obstaculizadores sociofamiliares, representan condiciones referidas a un nivel socioeconómico y cultural bajo y un rol parental educativo limitado que repercute en una menor valoración de la educación, un mayor interés por el estatus económico y la pérdida del estatus social del profesorado.

Los niños de a poco han perdido el interés en lograr un título o en lograr terminar un proceso como la básica y la media, porque ellos han visto en tíos, primos, familiares, en sus padres, amigos, que tienen séptimo, octavo o primero y ellos ganan mucho dinero, más que un profesor, entonces para ellos es irrelevante, es como un trámite venir al colegio (EI).

Consideran (b) obstaculizadores escolares, la presencia de una macro y micro política educativa que pone énfasis en los resultados y el desarrollo cognitivo más que en el socioemocional, que establece contenidos curriculares poco pertinentes y sobrecargan la función del profesorado y del estudiantado, asociado a una organización del tiempo escolar que impide la consideración de las necesidades de aprendizaje y desarrollo socioemocional.

Entonces yo, un poco, esto de regirnos por planes y programas que no conocen la realidad como decía R., del entorno en donde el niño está aprendiendo, ya, no se van a poder lograr cosas o el profesor va a estar un poco presionado a que el niño logre ese aprendizaje como sea, sin importarle lo que el niño esté sintiendo, si el niño durmió bien, si el niño se alimentó 
bien, si el niño tiene un espacio en su casa para poder estudiar, por qué, porque tenemos que regirnos por planes y programas (DGII; PI).

Con respecto a los facilitadores del aprendizaje y desarrollo socioemocional, visualizan un mínimo de estos. Básicamente consideran que los recursos de aprendizaje de tipo didáctico y tecnológicos favorecen la disposición al aprendizaje o motivación del estudiantado. Además, una parte explica que una institución educativa con un clima social escolar positivo y la formación permanente del profesorado en la temática facilitan el desarrollo socioemocional.

El aprendizaje con los materiales es excelente, porque yo soy una de las profesoras que me gusta que los niños no solamente con la pizarra y el plumón... actualmente ahora tenemos nosotros el laboratorio móvil, que los niños se lleven el computador a la sala, su mini computador, vamos al aula de recursos, ellos trabajan con su pizarra digital, hay material didáctico y muy buenos, pero a veces no sabemos sacarle provecho (EII).

Pienso yo que es importante también, ya que el marco institucional, las condiciones son muy adversas, es también tener uno una voluntad de informarse respecto de digamos ciertas tendencias. Yo tuve una experiencia... metiéndome en el tema de la inteligencia emocional (GDI; PII).

\section{El desarrollo socioemocional como proceso}

El desarrollo socioemocional del estudiantado en la escuela también es descrito como un proceso que se inicia al ingresar al establecimiento educativo, donde se evidencia un nivel evolutivo que sus docentes asocian con la influencia de la escuela, el grado escolar cursado, la maduración psicológica y biológica, y con estrategias de enseñanza adecuadas para dicho período.

Yo creo que el alumno, por ejemplo, el alumno nuestro, el alumno que llega a prekínder y aquí en la escuela sale de octavo, nosotros un poco, se moldea a nuestra manera, a nuestra forma de ser como comunidad educativa, por lo tanto al niño lo formamos de tal forma que le entregamos los valores para que él en octavo diga efectivamente yo estuve en la escuela X. y los profesores me entregaron estos valores (GDII; PIII).

\section{Etapa de la inmadurez socioemocional}

Para la mayoría de docentes, el estudiantado que cursa primer ciclo de enseñanza básica presenta un desarrollo socioemocional que se caracteriza por (a) otorgar un sentido lúdico al mundo y la escuela, por lo que la diversión es la prioridad y la enseñanza es vista como un juego; (b) una etapa de inexperiencia, inseguridad e ignorancia; (c) lo que se asocia a vulnerabilidad emocional y dependencia para la resolución de problemas interpersonales.

Desde lo anterior, el grupo participante considera que las estrategias de enseñanza adecuadas para este período implican educarle lúdicamente, además de velar por su integridad física y emocional.

El apego es más en básica, el apego es mucho mayor y es por lo mismo porque los primeros niveles hay menos profesores con ellos y después van pasando y tienen una diversidad de profesores y la presión a la que se van enfrentando ellos también (GDI; PVI).

¿Con quién me toca clases?, con la profesora, entonces no se me han perdido los niños, no tengo accidente escolar, a lo mejor tengo muchas falencias en otras cosas, pero si usted me pregunta, de primero a cuarto, los niños son para mí mis pollos y donde están ellos estoy yo (EII).

\section{Etapa de inicio de la madurez socioemocional}

Estudiantes que cursan segundo ciclo de educación básica inician este proceso con un menor sentido lúdico del mundo y la enseñanza, característica que tiende a desaparecer una vez egresan de octavo básico. Así mismo, 
el grupo participante considera que durante esta etapa el alumnado sucesivamente comienza a reservar sus problemas, incrementa el nivel de responsabilidad y confianza, y logra un mayor desarrollo de habilidades sociales para interactuar con sus docentes.

Acá en la escuela yo hago de primero a octavo, justamente hoy vino una niña y yo le decía: “cuando te conocí en primero eras muy tímida”. Ella era calladita, uno le preguntaba y uno tenía que acercarse casi al oído para decir lo que le pasaba, en cambio ahora ya no, ahora está en quinto y ya la veo mucho más desenvuelta, hasta hace bromas, me hizo bromas hoy (EVI).

Entonces yo creo que ahí, la pregunta que usted nos hace, puede ser relacionada a estos cambios también de ciclos educacionales. Ya el niño de $5^{\circ}$ y $6^{\circ}$ ya su cuerpo va cambiando, entonces son otros los intereses, las niñitas ya se empiezan a apilurar un poquitito ya, ya los varones están mirando para el lado, entonces son cambios generacionales, pero que también van acompañados de este cambio que, se podría decir estructural del aprendizaje, desde una tía, a tener un profesor y después a tener varios profesores (GDII, PIII).

\section{Etapa de crisis en el desarrollo socioemocional}

El grupo participante describe la transición hacia la enseñanza media y el ingreso a primer año de educación secundaria como un período en donde el desarrollo socioemocional del estudiantado se caracteriza por (a) una ausencia de sentido a lo que se enseña en el liceo, (b) lo que produce desinterés por aprender formalmente y (c) menor confianza y cercanía con el personal docente; también (d) se presenta un mayor apego con el grupo de amigos y amigas en búsqueda de apoyo emocional, por lo que (e) los problemas que les afectan quedan reservados a esta red de interacción.

Consideran que nuevamente se torna necesario hacer adecuaciones a la forma en que educan, para lo que deben acentuar el uso de herramientas que favorecen la superación de esta crisis. Básicamente, la estrategia está puesta en establecer conversaciones empáticas con sus estudiantes, a fin de destacar la necesidad de madurar emocionalmente, además de otorgarle un sentido a la enseñanza en función del logro académico futuro.

Claro, en cambio cuando los niños están en la enseñanza media, octavo básico o en enseñanza media, se ve reflejado de manera diferente, ellos ya prefieren guardarse dichos problemas, no son tanto de comentarios, se los comentan a su mejor amigo, claro que es notorio el cambio, es muy notorio, ya no se ve tanta cercanía entre el profesor y el alumno (EIV).

Pero en primero medio no es así, cuesta que el niño entienda que esto le va a servir para algo y se les dice: "como tú eres grande, si tú quieres estudiar necesitas aprender geometría", porque yo le hago geometría en primero medio, "necesitas hacer este tipo de cosas porque la PSU te lo va a pedir, o sea viene eso en la PSU", hay varios muy inteligentes pero como que no quieren aprender, les da lo mismo (EIII).

\section{Etapa de madurez socioemocional}

Desde el grupo participante, la transición de segundo a cuarto medio implica una fase de cambios significativos en el desarrollo socioemocional estudiantil. Básicamente, durante esta etapa se manifiesta (a) un mayor interés por la enseñanza que imparte la escuela, (b) asociado a la capacidad de reflexionar acerca de la importancia de la educación formal, (c) el otorgamiento de un sentido a esta misma y (d) un mayor respeto y confianza en el personal docente. Desde lo anterior, la enseñanza requiere de menores esfuerzos docentes.

Hay niños que están en tercero medio y cuando estaban en primero medio eran terribles y ahora pasan y me saludan, antes no me saludaban, y se arrepienten y dicen: "yo siempre fui desordenado con usted profesor" (EIII).

Un cuarto medio ya es más relajado, en mi caso los niños yo no les doy mayores instrucciones, sino que llego prácticamente a un acuerdo... ellos se sienten mayores, con mayor libertad, son los más grandes del colegio, así que claro que hay un cambio en la manera de entregar el contenido como ellos lo reciben (EIV). 


\section{Propuestas formativas para el aprendizaje y desarrollo socioemocional}

Se señala que el aprendizaje y desarrollo socioemocional del estudiantado es una tarea compleja que requiere de intervenciones de carácter sistémico y en donde la escuela tiene una función importante. Parte de estas propuestas incluyen el propio desarrollo socioemocional del profesorado y la gestación de competencias para instalar procesos formativos con sus estudiantes, para lo que se requiere de equipos de especialistas en la temática, que la aborden durante la formación inicial y continua. Otras propuestas formativas son las siguientes.

\section{La educación parental}

Consideran que la función educativa de los padres y madres se encuentra limitada, por lo que resulta necesario (a) fortalecer la relación con la familia, lo que podría redundar en una mejora de los lazos y apoyo en la labor que realiza la escuela. También sugieren (b) proporcionar a los padres y madres orientación a fin de que fortalezcan la relación que establecen con sus hijos o hijas y sean capaces de transmitir normas y reglas consistentes, que incrementen el desarrollo de la disciplina.

Bastante fácil, con la ayuda del establecimiento, involucrar a los padres, en las reuniones de apoderados, hacerles una pequeña charla instructiva, contarles: "mire, saben que sus niños ya están empezando a ver qué hacer con sus vidas, ustedes podrían contarles de logros, de comodidad en el trabajo, de aspectos más que nada espirituales más que económicos” (EI).

\section{Centradas en la estrategia pedagógica}

Explican que es necesario instalar estrategias pedagógicas que favorezcan el desarrollo socioemocional del estudiantado, que en términos generales, logren una coherencia entre las demandas sociales, lo que se enseña y la forma de evaluarlo; además de instalar un ambiente de aprendizaje de respeto, cordial, grato y atractivo, para lo que se requiere de docentes que cumplan un perfil docente apropiado.

Porque aquí hay profesores que no les gusta la modernidad, y no los culpo, porque es su forma de enseñar y también da resultado, pero por mi parte me gustaría que usaran la tecnología para que la enseñanza y el aprendizaje de ellos fuera más atractivo (EI).

Una de las estrategias es tener un ambiente adecuado, que ellos sientan acogedora la sala, el color como se las pinte, cómo está distribuido el mobiliario, dónde me siento yo (EII).

Además, se propone basar la enseñanza en un (a) aprendizaje práctico-vivencial, en donde el estudiantado deba reflexionar, plantear soluciones e implementarlas desde problemáticas reales y locales; (b) aprendizaje por modelado, en donde exalumnado de la institución concurra al establecimiento con la finalidad de relatar su experiencia de desarrollo personal y profesional, constituyéndose esto en un ejemplo; finalmente, (c) que docentes ejemplifiquen formas adaptativas de relacionarse con las demás personas, además de (d) incluir procesos de orientación hacia el estudiantado.

Hoy que me topé con ella y llegó tarde, siempre llega tarde esta niña y yo cuando llegan tarde, les digo que toquen la puerta y que digan: "buenos días profesor, ¿podemos pasar?", esa es la forma de saludar a los compañeros y a mí (EIV).

Hoy les contaba cuando yo era pequeña y ellos se reían y yo los veía que los tenía calladitos, y yo les decía: "yo también fui como ustedes, fui tan desordenada que mi señorita me pescaba de acá", y yo por eso que nunca hago eso porque a mí me dolía tanto mi oreja, y era tan conversadora, entonces las vivencias (EII). 


\section{Centrada en las macro y micropoliticas educativas}

Existe consenso en considerar necesario que las políticas educativas le otorguen una mayor importancia a la educación socioemocional del estudiantado. Explican que esta se encuentra relegada en un segundo plano, por lo que las escuelas no cuentan con estrategias sistemáticas y planificadas para este tipo de formación y, más bien, se fundamentan en esfuerzos personales y aislados.

No hay políticas institucionales respecto de eso, no hay políticas a nivel nacional y no hay políticas particulares respecto de eso, por lo tanto no, en este momento aunque yo quiera, aunque pudiera querer tener el tiempo, pudiera querer hacer tal cosa, son solamente esfuerzos individuales y finalmente son esfuerzos que pasan por el criterio de uno (GDI; PIII).

\section{La formación docente y el desarrollo socioemocional del alumnado}

Respecto de cómo se construyen las TS sobre el aprendizaje y desarrollo socioemocional, la mayoría de docentes identifica un déficit en su proceso formativo, situación que afecta su propio desarrollo socioemocional y la capacidad para promoverlo en sus estudiantes.

I: ¿Cómo ustedes evalúan o valoran por ejemplo que un alumno o en un grupo de curso, tienen buenas o malas habilidades socio-emocionales o son más maduros o menos maduros?

Al ojímetro más que nada, no hay una pauta, es como un sí parece que esto, que este es bueno, este es malo (EIII).

Es que ahí hay un tema importante porque nosotros estamos muy al debe en relación a lo que se está generando como conocimiento, por un lado y estamos muy al debe en la preparación como modelos (GDI; PIII).

No obstante lo anterior, identifican dos fuentes de formación que les permiten adquirir herramientas para promover el desarrollo socioemocional: experiencias formales e informales de aprendizaje, estas últimas ocurridas antes, durante y posteriormente a la formación inicial. Reconocen que durante su formación inicial existieron espacios formales de aprendizaje para la enseñanza de valores; sin embargo, las experiencias de mayor desarrollo surgieron en contextos informales de aprendizaje, donde destacan el autoaprendizaje, la crianza familiar y el currículo oculto.

Yo lo aprendí en mi casa ... en mi familia siempre la parte valórica fue importante, mi papá era muy estricto, cariñoso nunca fue, pero fue una persona muy estricta; mi mamá sumisa, pero correcta, mi mamá siempre con ganas de superación y siempre nos inculcó el camino correcto (EV).

Una de las experiencias que me marcó mucho fue trabajando 12 años con la pastoral ... y yo me di cuenta que los jóvenes buscan que se les escuche, ser escuchados y yo creo que es por la carencia de comunicación con la familia, entonces yo me he dado cuenta que los jóvenes y sobre todo los niños, les hace falta ser escuchados, eso me marcó (EVI).

\section{Discusión}

Con base en la función que se le asigna a las TS (Castro y Cárcamo, 2012; Catalán, 2010, 2011, 2016; Cuadra, 2009; Cuadra y Catalán, 2016; Cuadra et al., 2015; Flick, 2006; Janssens y Kelchtermans, 1997), es posible decir que los hallazgos encontrados aportan en comprensión respecto de cómo el personal docente explica y acciona en algún nivel sobre el aprendizaje y desarrollo socioemocional de sus estudiantes.

La estructura de las TS encontradas presenta asociaciones entre antecedentes y consecuentes, lo que permite asumir que poseen un nivel de elaboración que le otorga al personal docente la capacidad de explicar este fenómeno y, desde allí, regular en algún grado sus prácticas pedagógicas, orientándolas al desarrollo socioemocional del estudiantado, aunque esto no implica la presencia de enunciados profundos o alternativos (Castro y Cárcamo, 2012; Catalán, 2010), sino que más bien suponen explicaciones sencillas, situación que desde estas personas participantes es advertido en el reconocimiento de la existencia de limitaciones en su proceso formativo para enfrentar esta tarea, lo que ya había sido reportado en otras investigaciones (De Leyva, 
2015; Vivas et al., 2010). Al respecto, Ochoa y Peiró (2012b) advierten de debilidades en la formación del profesorado para la educación socioemocional, situación que otros estudios (Díaz, 2014; López de Lérida, 2009) explican basándose en una formación principalmente centrada en el desarrollo de competencias de pedagogía, para el desarrollo cognitivo de su futuro alumnado, en desmedro de la adquisición de capacidades para la educación socioemocional.

Desde el programa de investigación de TS, la simplicidad de este tipo de representaciones (Cuadra, 2009; Waeytens, Lens y Vandenberghe, 2002) o menor elaboración (Castro y Cárcamo, 2012; Catalán, 2010, 2016), implica que la persona representa un fenómeno con enunciados que tienen una estructura argumentativa, que no logra una red compleja de relaciones entre antecedentes y consecuentes, lo que lingüísticamente se observa como "una explicación sencilla". Esto resulta problemático cuando el fenómeno a explicar, desde el saber científico técnico, requiere del domino de modelos teóricos que relacionan múltiples variables para comprenderlo y abordarlo. Precisamente la educación socioemocional parece ser un fenómeno complejo y difícil de delimitar (García, 2012).

Lo anterior ha sido señalado como una condición que podría limitar las prácticas pedagógicas (Cuadra et al., 2017). Considerando la implicancia que tiene la educación y desarrollo socioemocional (Berger et al., 2011; Berger et al., 2009; Cohen, 2003; Díaz, 2014; García, 2012) y desde el planteamiento anterior, se torna necesario que el personal docente logre desarrollar explicaciones de mayor elaboración, a propósito de la complejidad de la temática y la diversidad de posiciones teóricas existentes (Abarca, 2003). El poseer explicaciones más profundas y alternativas permite instalar procesos reflexivos en estos grupos profesionales, orientados a la toma de decisiones frente a la educación socioemocional, pues se considera que la escuela es un escenario social complejo (Bravo y Herrera, 2011), que enfrenta problemáticas que dificultan la instalación de una convivencia escolar nutritiva (Jares, 2006), frente a lo cual las decisiones profesionales se tornan fundamentales.

Esto representa un sustento que contribuye en la comprensión de la necesidad de fortalecimiento de la formación docente en la enseñanza y desarrollo socioemocional del estudiantado (Díaz, 2014; López de Lérida, 2009; Ochoa y Peiró, 2012b), además de dar luces acerca de cómo implementar estos procesos de mejora. Uno de estos elementos se relaciona precisamente con las experiencias formativas del profesorado para la educación socioemocional, que desde estos resultados incluyen aquellas de tipo formales e informales, hallazgos que se corresponden con otras investigaciones que han apuntado a responder cómo es que el personal docente explica su formación profesional (Cuadra y Catalán, 2016; Hindin, 2010). Al respecto, la sugerencia formativa se orienta a identificar (Helmke, 2009; Wahl, 2002), explicitar, promover el autoconocimiento (Linsner aus Düsseldorf, 2009) y reflexión sobre las TS que sustentan las prácticas pedagógicas (Brighenti y Catalán, 2014; Catalán, 2010, 2011, 2016; Castro et al., 2012; Castro, Krause y Frisancho, 2015; Cuadra et al., 2015; Seebauer, 2009), en este caso, las que se orientan a la educación socioemocional.

Implica que el profesorado, mediante procesos formativos formales y con base en la estrategia mencionada anteriormente, logre integrar el saber científico técnico, con sus teorías profesionales y teorías subjetivas de manera reflexiva, para llegar a desarrollar un conocimiento profesional idóneo, que le permita abordar un problema complejo, como lo es la educación socioemocional.

El contenido de las TS de estos grupos docentes permite representar el aprendizaje y desarrollo socioemocional en contexto escolar, como la formación valórica, de hábitos y habilidades sociales del estudiantado, que se expresa en perseverancia para lograr metas, respeto en las relaciones interpersonales, hábitos de cortesía, honestidad, disciplina y buen desempeño en las tareas académicas; y se vincula significativamente con el otorgamiento de un sentido a la vida y una condición necesaria para generar el aprendizaje del currículo. Esta forma de concebir el aprendizaje y desarrollo socioemocional es consistente con lo que actualmente algunos estudios proponen sobre la temática, en donde se destaca el amplio valor que tiene y su relación estrecha con el desarrollo del estudiantado (García, 2012), la capacidad de este mismo para 
accionar constructivamente en el medio (Berger et al., 2009; Cohen, 2003) y, en definitiva, lograr el bienestar personal y el de otros seres (Berger et al., 2011). En esta misma línea, parte de los hallazgos obtenidos por Toro y Berger (2012) en docentes de una comuna de Santiago de Chile, quienes muestran el grado de importancia que le otorgan a la educación socioemocional, llegando incluso a considerarla como una relación parental secundaria establecida con sus estudiantes y que desde otros modelos teóricos se ha venido conceptualizando como una relación de apego entre profesorado y alumnado, con características similares a la establecida entre padres y madres y su progenie (Maldonado y Carrillo, 2006).

Desde esto, parece ser que este sentido subjetivo cumple el primer requisito para la educación socioemocional: orienta la acción docente o la dispone hacia la instalación de prácticas pedagógicas que permitan desarrollar esta competencia en sus estudiantes. Esta presunción cobra relevancia si se considera que en otras investigaciones de TS docentes se han encontrado unas de tipo epistemológicas (Cuadra, 2016), que como parte de su estructura y función evalúan el conocimiento a impartir, pudiendo actuar como inhibidoras de los procesos de cambio cuando se le otorga un bajo valor a una temática de enseñanza (Cuadra et al., 2015).

En cuanto a cómo explica el personal docente la formación y desarrollo socioemocional de sus estudiantes, se identifican factores sociales, familiares, individuales y escolares que afectan este proceso y dentro de estos mismos, facilitadores y obstaculizadores, estos últimos los que, a juicio de docentes, se encuentran más presentes. Otras investigaciones en docentes han dado cuenta de TS que incluyen en su estructura argumentativa una mayor cantidad de obstaculizadores de prácticas pedagógicas, lo que ha sido relacionado con la agentividad docente y el sentido de eficacia (Castro y Cárcamo, 2012; Cuadra, 2009; Volante, Tapia y Müller, 2005). En estos hallazgos, una mayor presencia de obstaculizadores para implementar la educación socioemocional podría conllevar a una inhibición de esta acción o a su limitación, en este caso, atribuible por docentes a las condiciones socioculturales de las familias de sus estudiantes y a la presencia de macro y micropolíticas que descuidan lo emocional y se centran en lo cognitivo; además de focalizarse en los resultados educativos utilizando medidas de presión que hacen sentir al personal docente una insuficiencia del tiempo escolar, que incluso afecta su salud laboral. Este resultado se ha encontrado con mucha frecuencia en esta profesión (Castro y Cárcamo, 2012; Cuadra et al., 2015; Universidad Alberto Hurtado, 2012; Volante y Müller, 2006), lo que indica la urgencia de considerarlo a la hora de establecer medidas de mejora. Lo anterior cobra sentido al resumir el pensamiento docente frente a la educación socioemocional, en una interpretación de segundo orden y desde estos hallazgos: (a) le otorga una alta importancia y logra tener algún nivel de explicación de esta misma; (b) pero considera que existen condiciones familiares, escolares, sociales y políticas que limitan considerablemente su implementación, además de (c) limitaciones en su formación profesional para abordarla.

Un último hallazgo que queremos destacar devela que el personal docente también comprende el desarrollo socioemocional del estudiantado como proceso, lo que podría complementar los modelos evolutivos de desarrollo de esta área estudiantil, al considerar que este grupo profesional se constituye en una figura significativa para el alumnado y que el tiempo escolar exige que sus estudiantes permanezcan cada vez más en las escuelas (Martinic, 2015), lo que torna protagónica la mirada docente al respecto. Desde estos resultados, el desarrollo socioemocional del estudiantado se explica con base en cuatro etapas evolutivas, para las que se requiere la instalación de determinadas prácticas pedagógicas que buscan adaptarse a las características del alumnado. Este planteamiento está en línea con lo señalado por Bisquerra (2006), respecto de que la educación socioemocional es un proceso formativo de toda la vida y se produce desde un enfoque de ciclo vital; y se hace parte de los objetivos que se ha propuesto el Grupo de Investigación Psicopedagógica de la Universidad de Barcelona (GROP), básicamente de la necesidad de secuenciar la adquisición de competencias socioemocionales del estudiantado a lo largo del currículo (De Leyva, 2015). Desde esto, parece pertinente sugerir investigaciones que permitan profundizar respecto de cómo es que transcurre el desarrollo socioemocional del estudiantado, en función del ciclo escolar, las tareas propias que le exige el contexto educativo y las prácticas pedagógicas que requiere adecuar para este desarrollo evolutivo. 
La Figura 1 muestra cómo se relacionan las teorías subjetivas encontradas. Representa un modelo comprensivo que posibilita comprender el aprendizaje y desarrollo socioemocional basado en estas cuatro etapas evolutivas que transcurren en el contexto escolar y que se interrelacionan con el significado subjetivo que el personal docente tiene de la temática y multifactores que pueden actuar como facilitadores u obstaculizadores de este proceso, además de medidas remediales identificadas por el personal docente; TS del profesorado que surgen fundamentalmente desde experiencias formales e informales de aprendizaje y antes, durante y posteriormente a la formación inicial.

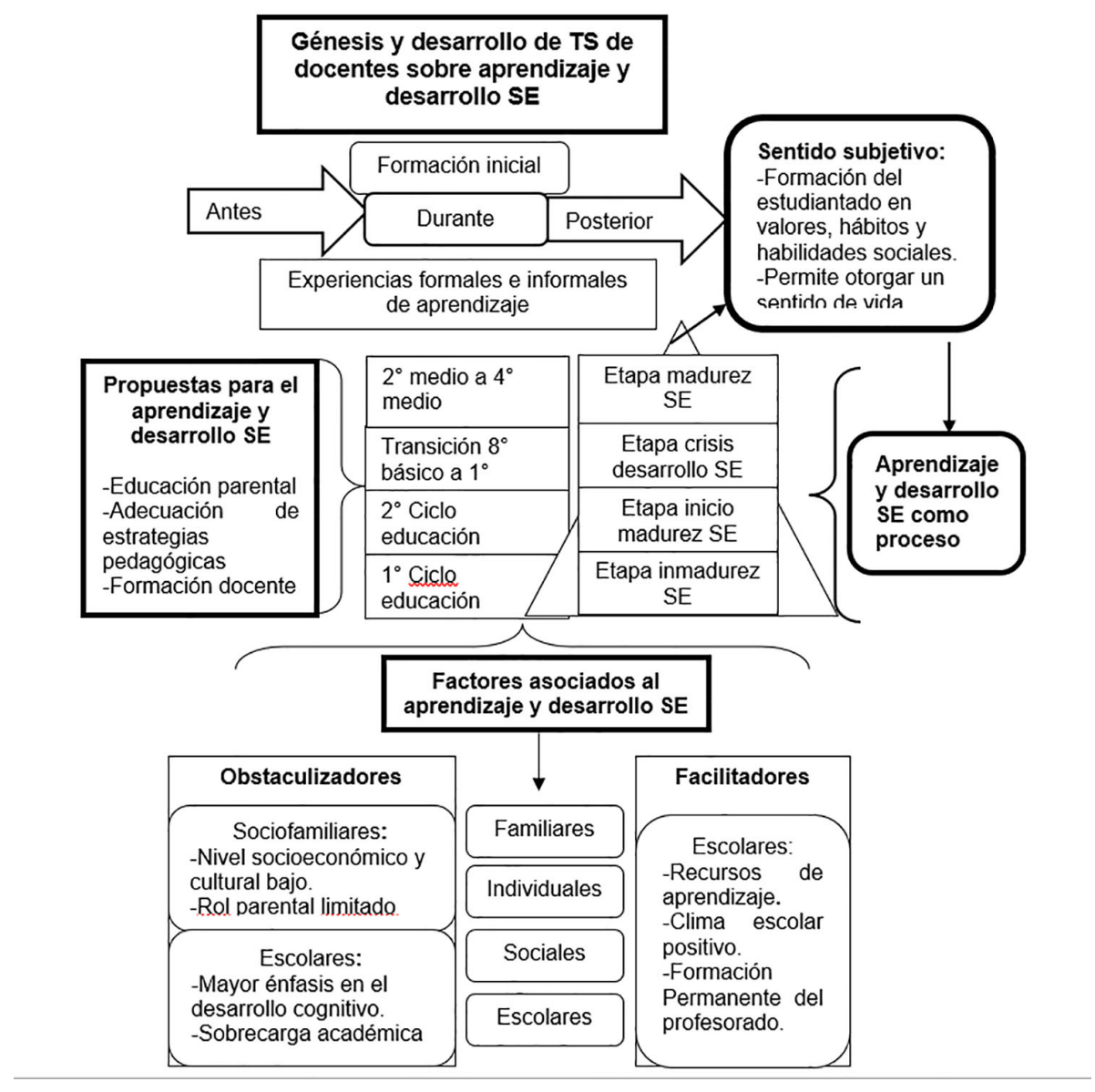

FIGURA 1

Codificación selectiva. Modelo comprensivo del aprendizaje y desarrollo socioemocional del estudiantado. $\mathrm{SE}=$ socioemocional; $\mathrm{TS}=$ teoría subjetiva.

Con respecto al método, parece pertinente incentivar la realización de investigaciones sobre el tema que permitan prolongar la descripción del desarrollo socioemocional a estudiantes de educación superior, a propósito de que el modelo de formación por competencias ha cobrado importancia durante el último tiempo en este nivel de enseñanza y las competencias socioemocionales son claves del proceso formativo (Villarroel y Bruna, 2014).

Se sugiere, con ello, la importancia de articular los saberes pedagógicos y las competencias terminales en la formación de las futuras generaciones docentes (Leguizamon, 2014); atendiendo la brecha formativa que por lo tanto- ocurre entre estos saberes en el ámbito de lo socioemocional y la innovación curricular (Lería, Salgado y Sasso, 2018); todo ello dentro del reconocido movimiento por la profesionalización de la enseñanza y la generación de relaciones más simétricas, sistémicas e interactivas entre la teoría y la práctica (Cornejo, 2014). 
En cuanto a los instrumentos de recolección de información, la entrevista episódica (Catalán 2010; Castro y Cárcamo, 2012; Flick, 2006) y el grupo de discusión basado en episodios biográficos como medio de producción de TS se tornan una herramienta eficiente y facilitadora de la elicitación de TS, en la medida que les permite a las personas participantes contextualizar el origen de sus representaciones subjetivas (Cuadra, 2016).

Finalmente, queda señalar la necesidad de promover investigaciones orientadas a la comprensión del cambio de las TS de docentes, en este caso, referidas a la educación socioemocional del estudiantado, lo que permitiría complementar el estado del arte para el complejo desafío de producir el cambio en educación (Flores \& De La Torre, 2010) y dar mayor sustento al programa de investigación de TS, como paradigma emergente de investigación educativa (Cuadra et al., 2017).

\section{REFERENCIAS}

Abarca, M. (2003). La educación emocional en la educación primaria: Currículo y práctica (Tesis doctoral). Universitat de Barcelona.

Berger, C., Alcalay, L., Torretti, A., \& Milicic, N. (2011). Socio-emotional well-being and academic achievement: Evidence from a multilevel aproach [Bienestar socio-emocional y desempeño académico. Evidencia desde un enfoque multinivel]. Psicología: Reflexão e Crítica, 24(2), 344-351.

Berger, C. Milicic, N., Alcalay, L., Torretti, A., y Arab, M. (2009). Bienestar socio-emocional en contextos escolares: La percepción de estudiantes chilenos. Estudios Sobre Educación, 17, 21-43.

Bisquerra, R. (2006). Orientación psicopedagógica y educación emocional. Estudios sobre Educación, 11, 9-25.

Bravo, I., y Herrera, L. (2011). Convivencia escolar en educación primaria. Las habilidades sociales del alumnado como variable moduladora. Dedica. Revista de Educação e Humanidades, 1, 173-202. Recuperado de http:// dialnet.unirioja.es/revista/15495/A/2011

Brighenti, M., y Catalán, J. (2014). Teorías subjetivas de profesores en reuniones de trabajo: Un estudio descriptivointerpretativo. Revista Quadrimestral da Associação Brasileira de Psicologia Escolar e Educacional, SP, 18(1), 151-159.

Bruns, B., y Luque, J. (2014). Docentes excelentes: Cómo mejorar el aprendizaje en América Latina y el Caribe (resumen). Washington, DC: Banco Mundial.

Burgos, N. (2011). Investigación cualitativa. Miradas desde el trabajo social. Buenos Aires: Espacio editorial.

Castro, P. (2006). Sujetos en la política educacional chilena de transversalidad. Psykhe, 15(2), 37-43. doi:10.4067/ S0718-222820060002000310

Castro, P., Agüero, C., Barraza, A., Escobar, G., y Jorquera, J. (2012). Disposición a la reflexión colectiva sistemática en docentes de un centro educativo de Chile. Revista Mexicana de Investigación Educativa, 17(53), 573-591.

Castro, P., y Cárcamo, R. (2012). Cambio de teorías subjetivas de profesores respecto a la educación en valores. Subjetividad y Procesos Cognitivos, 16(1), 17-42.

Castro, P., General, F., Jofré, R., Sáez, N., Vega, A., y Bortoluzzi, M. (2012). Teorías subjetivas de profesores sobre la motivación y sus expectativas de éxito y fracaso escolar. Educ. Rev., Curitiba, 46(4), 159-172.

Castro, P., Krause, M., y Frisancho, S. (2015). Teoría del cambio subjetivo: Aportes desde un estudio cualitativo con profesores. Revista Colombiana de Psicología, 24(2), 363-379.

Catalán, J. (2010). Teorías subjetivas. Aspectos teóricos y prácticos. La Serena, Chile: Editorial Universidad de La Serena.

Catalán, J. (2011). Del pensamiento al conocimiento profesional del profesor. En J. Catalán (Ed.), Psicología educacional. Proponiendo rumbos, problemáticas y aportaciones (pp. 187-215). La Serena: Editorial Universidad de La Serena.

Catalán, J. (2016). Hacia la formulación de una teoría general de las teorías subjetivas. Psicoperspectivas. Individuo y Sociedad, 15(1), 53-65. doi: 10.5027/PSICOPERSPECTIVAS-VOL15- ISSUE1-FULLTEXT-739 
Cohen, J. (2003). La inteligencia emocional en el aula: Proyectos, estrategias e ideas. Buenos Aires: Troquel.

Cornejo, J. (2014). Prácticas profesionales durante la formación inicial docente: Análisis y optimización de sus aportes a los que aprenden y a los que enseñan a aprender "a enseñar". Estudios Pedagógicos, 40(Especial), 239-256.

Cuadra, D. (2009). Teorías subjetivas en docentes de una escuela de bajo rendimiento, sobre la enseñanza y el aprendizaje del alumno. Revista mexicana de investigación educativa, 14(42), 939-967.

Cuadra, D. (2016). Cambio representacional en los padres: Reconstrucción de teorías subjetivas de la prevención del consumo de drogas en los hijos e hijas. Revista Estudios Pedagógicos, 42(1), 283-298. Recuperado de https:// dx.doi.org/10.4067/S0718-07052016000100018

Cuadra, D., Castro, P. J., Vystrčilová, P., y Jancic Mogliacci, R. (2017). A review of research on teachers' subjective theories: contributions to the study of teacher education [Una revisión de investigaciones de teorías subjetivas de profesores: Contribuciones al estudio de la formación del profesor]. Psychology and Education an Interdisciplinary Journal, 54(3), 1-22.

Cuadra, D. y Catalán, J. (2016). Teorías subjetivas en profesores y su formación profesional. Revista Brasileira de Educação, 21(65), 299-324. Recuperado de http://www.scielo.br/pdf/rbedu/v21n65/1413-2478rbedu-21-65-0299.pdf

Cuadra, D., Jorquera, R., y Pérez, M. (2015). Las teorías subjetivas del profesor acerca de su salud laboral: Implicancias en la promoción de la salud preventiva del trabajo docente. Ciencia \& Trabajo, 17(52), 1-6.

De Leyva, Y. (2015). Las competencias socioemocionales de los docentes de educación secundaria de la comunidad de Madrid en contextos de acción tutorial (Tesis de maestría), Universidad Complutense de Madrid, Madrid. Recuperado de http://eprints.sim.ucm.es/36129/

Díaz, T. (2014). El desarrollo de competencias socioemocionales y su evaluación como elementos clave en los planes de formación docente. Algunas conclusiones derivadas de la evaluación SIMCE 2011. Revista Iberoamericana de Educación, 64, 73-98.

Donovan, D., Borda, E., Hanley, D., \& Landel, C. (2015). Participation in a multi-institutional curriculum development project changed science faculty knowledge and beliefs about teaching science [Participación en un proyecto multiinstitucional de desarrollo del curriculum y el cambio generado en las creencias y conocimientos acerca de la enseñanza de la ciencia]. Journal of Science Teacher Education, 26(2), 193-216. Doi: 10.1007/ s10972-014-9414-z

Flick, U. (2006). An introduction to qualitative research [Introducción a la investigación cualitativa] (3a ed.). London: Sage.

Flores, E., y De La Torre, M. (2010). La problemática de la Investigación sobre cambio educativo. Revista mexicana de investigación educativa, 15(47), 1017-1023. Recuperado de http://www.scielo.org.mx/scielo.php? script=sci_arttext\&pid=S1405-66662010000400002\&lng=es\&tlng=es.

Friesen, S. (2009). What did you do in school today? Teaching Effectiveness: A Framework and Rubric [¿Qué hiciste hoy en la escuela?. Enseñanza efectiva. Un marco y matriz de valoración]. Toronto: Education Association.

Fullan, M. (2002). Los nuevos significados del cambio en educación. Barcelona: Octaedro.

García,J. (2012). La educación emocional, su importancia en el proceso de aprendizaje. Revista Educación, 36(1), 1-24.

García, J., y Ballesteros, B. (2003). La formación del profesorado a través de los sistemas educativos a distancia. La evaluación del programa de la UNED. Revista de investigación educativa, 21(1), 47-65. Recuperado de revistas.um.es/rie/article/viewFile/109531/104121

Groeben, N., \& Scheele, B. (2000). Dialogue-hermeneutic method and the "research program subjective theories" [9 paragraphs] [El método dialógico-hermenéutico y el programa de investigación de teorías subjetivas]. Forum Qualitative Sozialforschung / Forum: Qualitative Social Research, 2(1). Recuperado de http://nbnresolving.de/urn:nbn:de:0114-fqs0002105.

Helmke, A. (2009). Unterrichtsqualität und Lehrerprofessionalität. Diagnose, Evaluation und Verbesserung des Unterrichts [Calidad de la enseñanza y profesionalización docente. DIagnóstico, Evaluación y mejora de la enseñanza]. Seelze: Klett Kallmeyer. 
Hindin, A. (2010). Linking Home and School: Teacher Candidates' Beliefs and Experiences [La interrelación familiaescuela: Creencias y experiencias de futuros docentes]. The School Community Journal, 20(2), 73-90.

Janssens, S., \& Kelchtermans, G. (1997). Subjective Theories and Professional Self of Beginning Teachers [Teorías subjetivas y el autoconcepto profesional de docentes principiantes]. Paper presented at the Annual Meeting of the American Educational Research Association (Chicago, IL, March 24-28). Recuperado de http:// files.eric.ed.gov/fulltext/ED408252.pdf.

Jares, X. (2006). Pedagogía de la convivencia. Barcelona: Graó.

Kington, A. (2012). Narratives of variation in teacher-pupil relationships a cross a career [Narrativas y variaciones discursivas de la relación profesor-alumno a lo largo de la carrera]. European Journal of Educational Estudies, 4(2), 189-201.

Leguizamon, G. (2014). La construcción de saberes pedagógicos en la formación del profesorado. REICE, Revista Latinoamericana sobre Calidad, Eficacia y Cambio en Educación, 12(1), 35-54. Recuperado de https:// revistas.uam.es/index.php/reice/article/view/2863/3080

Lería, F. J., Salgado, J. A. y Sasso, P. (2018). Sentido subjetivo de educadoras de párvulos en el uso e impacto del silencio en el aprendizaje. REICE. Revista Latinoamericana sobre Calidad, Eficacia y Cambio en Educación, 16(2), 77-97. Recuperado de https:/doi.org/10.15366/reice2018.16.2.005

Linsner aus Düsseldorf, M. (2009). Prototypische routinen von lehrkräften im ungang mit unterrichtseinstiegen, experimenten und schülervorstellungen im biologieunterricht [Rutinas prototípicas de los maestros al inicio de clase: Experimentos y preconcepciones de los alumnos en la enseñanza de la biología.]. (InauguralDissertation zur Erlangung des Doktorgrades, Universität Duisburg-Essen, Duisburg-Essen). Recuperado de http://duepublico.uni-duisburg-essen.de/servlets/DocumentServlet?id=27202

López de Lérida, S. (2009). Las competencias docentes (Monografía inédita). Santiago: Programa Doctorado Universidad Católica.

Maldonado, C., \& Carrillo, S. (2006). Teaching with affection: characteristics and determinant factors of quality in teacherstudent relationships [Enseñanza con afecto: Características y factores determinantes de la calidad en las relaciones profesor-estudiante]. Journal Childhood Adolescent and Family, 1(1), 39-60.

Martinic, S. (2015). El tiempo y el aprendizaje escolar. La experiencia de la extensión de la jornada escolar en Chile. Revista Brasileira de Educação, 20(61), 479-499.

Ochoa, A., y Peiró, S. (2012a). El quehacer docente y la educación en valores. Tesis, 13(3), 28-48.

Ochoa, A., y Peiró, S. (2012b). La educación en valores en la formación incial de los profesores de educación básica en México. REIFOP, 15(1), 157-164.

Ramírez, I. (2011). El compromiso ético del docente. Revista Iberoamericana de Educación, 55(2), 2-6.

Retuert, G., y Castro, P. (2017). Teorías subjetivas de profesores acerca de su rol en la construcción de la convivencia escolar. Polis, 46. Recuperado de http://journals.openedition.org/polis/12395

Ruiz, O. (1999). Metodología de la investigación cualitativa. Bilbao: Universidad de Deusto.

Salgado, J. (2009). Pensamiento del profesor acerca del éxito o fracaso de su respectiva unidad educativa. Revista Iberoamericana sobre Calidad, Eficacia y Cambio en Educación, 7(3), 47-68.

Sandoval, M. (2014). Convivencia y clima escolar: Claves de la gestión del conocimiento. Última Década, 22(41), 153-178. Recuperado de http://dx.doi.org/10.4067/S0718-22362014000200007

Seebauer, R. (2009). The modification of subjective theories with Viennese teacher trainees: results from a two-year study between the first and the fourth semester of study [El cambio de teorías subjetivas en profesores vieneses en formación: Resultados de un estudio de dos años realizado entre el primer y cuarto semestre de estudio]. Orbis scholae, 3(2), 63-75.

Stake, R. (1999). Investigación con estudio de casos. Madrid: Ediciones Morata.

Strauss, A., y Corbin, J. (2002). Bases de la investigación cualitativa. Técnicas y procedimientos para desarrollar la teoría fundamentada. Antioquia, Colombia: Editorial Universidad de Antioquia. Primera Edición en Español.

Taylor, S., y Bogdan, R. (1987). Introducción a los métodos cualitativos de investigación. Barcelona: Paidós. 
Toro, L., y Berger, C. (2012). Percepción de profesores jefes de quinto y sexto básico de su rol en el aprendizaje socioemocional de los estudiantes. Revista Electrónica Diálogos Educativos, 12(23), 16-37.

Universidad Alberto Hurtado. (2012). Procesos de enseñanza - aprendizaje desde la perspectiva de los profesores en Chile (Informe de investigación). Santiago, Chile.

Villarroel, V., y Bruna, D. (2014). Reflexiones en torno a las competencias genéricas en educación superior: Un desafío pendiente. Psicoperspectivas, 13(1), 23-34.

Vivas, M., Chacón, M., y Chacón, Ed. (2010). Competencias socioemocionales autopercibidas por los futuros docentes. Educere, 14(48), 137-146.

Volante, P., y Müller, M. (2006). Cambios en el discurso sobre resultados de aprendizaje en escuelas de bajo rendimiento y alta vulnerabilidad social. Rev. Pensamiento Educativo, 39(2), 205-224.

Volante, P., Tapia, O., y Müller, M. (2005). Alineamiento y transferencia de control para el mejoramiento de aprendizajes. Bol. Invest. Educ., 20(2), 289-304.

Von Reininghaus, G., Castro, P., y Frisancho, S. (2013). School Violence: Subjective theories of academic advisory board members from six chilean schools [Violencia en la escuela: Teorías subjetivas de docentes directivos de seis escuelas chilenas]. Interdisciplinaria, 30(2), 219-234.

Waeytens, K., Lens, W., \& Vandenberghe, R. (2002). 'Learning to learn': teachers' conceptions of their supporting role [Aprendiendo a aprender: Concepciones de profesores acerca de su rol de apoyo]. Learning and Instruction, 12(3), 305-322. Recuperado de http://www.journals.elsevier.com/learning-and-instruction

Wahl, D. (2002). Veränderung subjektiver Theorien durch Tele-Learning? [¿Cambian las teorías subjetivas con la educación a distancia?] In Mutzeck, Wolfgang; Schlee, Jörg; Wahl, Diethelm (Hrsg.), Psychologie der Veränderung. Subjektive Theorien als Zentrum nachhaltiger Modifikationsprozesse [Psicología del cambio. Teorías subjetivas como centro de procesos de cambio sustentable] (pp. 10-21). Weinheim, Basel: Beltz.

\section{Notas}

[1] Esta investigación fue financiada por la Universidad de Atacama, a través del Fondo Concursable Dirección de Investigación, DIUDA. Proyecto DIUDA 15/12 22281, año 2015-2016.

[6] $\mathrm{GD}=$ grupo de discusión; $\mathrm{E}=$ entrevista; $\mathrm{I}=$ investigador; $\mathrm{P}=$ participante

\section{BY-NC-ND}

